\title{
Barchan dunes in two dimensions: Experimental tests for minimal models
}

\author{
Christopher Groh, ${ }^{1}$ Andreas Wierschem, ${ }^{2}$ Nuri Aksel, ${ }^{2}$ Ingo Rehberg, ${ }^{1}$ and Christof A. Kruelle ${ }^{1}$ \\ ${ }^{1}$ Experimentalphysik V, Universität Bayreuth, D-95440 Bayreuth, Germany \\ ${ }^{2}$ Technische Mechanik und Strömungsmechanik, Universität Bayreuth, D-95440 Bayreuth, Germany \\ (Received 24 December 2007; revised manuscript received 21 May 2008; published 15 August 2008)
}

\begin{abstract}
A well-defined two-dimensional single barchan dune under the force of a shearing water flow is investigated experimentally. From an initially prepared triangular heap a rapid relaxation to a steady-state solution is observed with constant mass, shape, and velocity. This attractor exhibits all characteristic features of barchan dunes found in nature, namely a gently inclined windward side, crest, brink, and steep lee face. The relaxation time towards the steady state increases with mass. For small dunes we find significant deviations from a fixed height-length aspect ratio. As predicted by recent theoretical models, the migration velocity scales reciprocal to the length of the dune.
\end{abstract}

DOI: 10.1103/PhysRevE.78.021304

PACS number(s): 45.70.- $\mathrm{n}$

The global climate change is one of the greatest problems of mankind for the next decades or even centuries [1]. One major result of the global warming is the intensification of the desertification process in arid and semiarid countries. Desertification, i.e., the degradation of productive land such as oases in the desert or the aggradation of inshore shallow waters, is an inexorable conflict between natural phenomena and human interests.

During the past decades, desert dune research has changed significantly from descriptive studies of dune morphology [2-4] to investigations more concerned with the microscopic processes of sediment transport [5] and the dynamics of sand-fluid interactions [6-8]. A recent review about this vast field of research is provided by Livingstone et al. [9].

Since first-principle equations for the dynamics of granular materials - with the same relevance as the Navier-Stokes equation in hydrodynamics - are still not available, simplified phenomenological, so-called "minimal models" have been proposed recently by several groups [10-15].

The favored test object is the barchan dune, a highly mobile crescent-shaped dune, which is found in unidirectional wind fields on bedrock not entirely covered with sand. Topographical profiles of real barchans from several field studies in Southern Morocco [10,11,16,17], as well as sand-flux data from wind tunnel experiments [18], and even from the surface of Mars [19], have been used to determinate the model parameters.

The often quoted observation that dunes migrate with a speed inversely proportional to their height $H$, originally suggested by Bagnold [20], has been questioned recently by Kroy and co-workers [12,21]. Based on a continuum model for the dynamics of barchan dunes, they predict that the migration velocity $v$ scales rather with the dunes length $L$ according to $v \sim L^{-1}$, at least for moderate winds and small dunes [12,21]. They also find discrepancies with recently published data on submarine dunes on a laboratory scale $[21,22]$ and suggested that further quantitative confirmations of the competing scaling laws $[12,14,20]$ are desirable. Since presently available field data are not accurate enough to clearly distinguish between $v \sim H^{-1}, v \sim\left(H+H_{0}\right)^{-1}$, or $v$ $\sim L^{-1}$, we put these contradicting scaling predictions to the test by measuring the dependence of the migration velocity on both the experimentally determined height and length of barchan dunes, generated under controlled laboratory conditions.

Therefore we study downsized replicas of natural barchan dunes in the laboratory, which are expected to have similar properties as the real ones. We have chosen water as driving medium instead of air because in water the barchan dunes exist in a convenient size for observation. A detailed explanation of these scaling properties is given in Refs. $[14,19,22,23]$. However, the validity of the "minimal transport model" underwater has been challenged by several authors [19,24-27]. In any case a convenient feature is that the time scale becomes smaller, which makes the experiments easier to handle. For example, the typical time for a barchan to travel over its own length is of the order of one year for a small $3 \mathrm{~m}$ high aeolian dune, while the corresponding $3 \mathrm{~cm}$ high aquatic dunes such as the one shown in Fig. 1 need only about $15 \mathrm{~s}$.

Former experiments in the laboratory with water involve three-dimensional barchan dunes [22,28,29]. For a direct comparison with theoretical models we construct a setup consisting of a narrow channel, which is intrinsically two dimensional.

Our experimental setup is shown in Fig. 2. It consists mainly of a flow tube machined from perspex which is filled

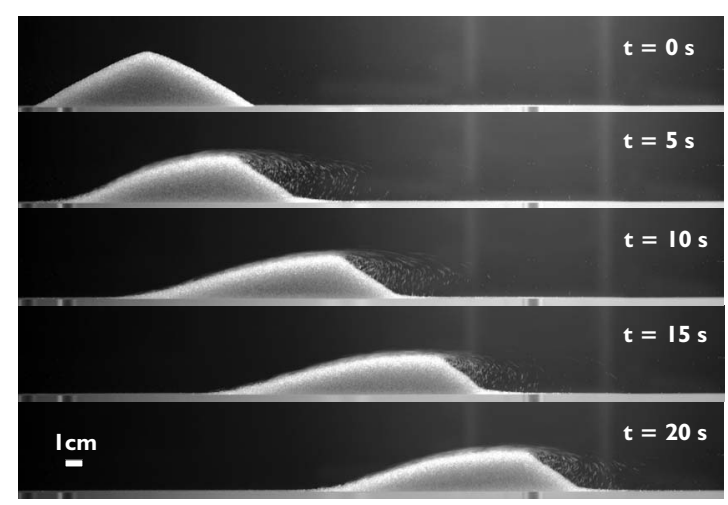

FIG. 1. Temporal evolution of a developing two-dimensional barchan dune subject to unidirectional water flow $(\operatorname{Re}=27000, m$ $=13 \mathrm{~g})$. 


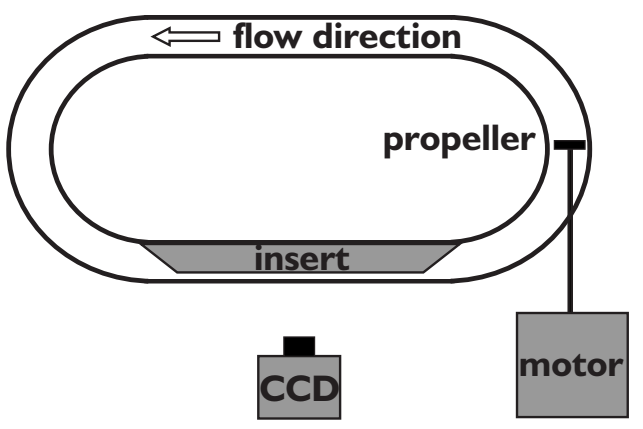

FIG. 2. Scheme of the experimental setup (top view).

with distilled water. The straight part has a length of $600 \mathrm{~mm}$. The curves have an outer diameter of $500 \mathrm{~mm}$ and an inner diameter of $400 \mathrm{~mm}$. The width of the channel results in $50 \mathrm{~mm}$ and its height is $60 \mathrm{~mm}$. A lid made of perspex is fixed at the top to close the channel. In the right curve a propeller with a diameter of $45 \mathrm{~mm}$ is installed. The propeller is driven by a motor with a shaft which is placed in the middle of the channel profile. The flow direction is counterclockwise.

The measurements are made with a charge-coupled device (CCD) camera (Lumenera Lw11059) which is placed in front of the straight part of the channel. The camera has a horizontal resolution of $4008 \times 2672$ pixels and a maximum frame rate of $5 \mathrm{fps}$. A black plastic insert is used to limit the width of the section of measurements to approximate twodimensional conditions. The insert constricts the channel to a width of $6 \mathrm{~mm}$. This leads to an aspect ratio of width to height of 10 .

After the flow tube is filled with distilled water, a funnel with a $6 \mathrm{~mm}$ long slit is used to pour glass beads into the channel. The experiments always start with the triangular heap shown in Fig. 1. The glass beads have a diameter ranging between $560-600 \mu \mathrm{m}$ and a density of $\rho=2.5 \mathrm{~g} / \mathrm{cm}^{3}$. The different sizes of the barchan dunes are selected by weighing different amounts of the glass beads. The selected masses $m$ amount to $2.17 \mathrm{~g}, 3.25 \mathrm{~g}, 6.5 \mathrm{~g}, 9.75 \mathrm{~g}, 13 \mathrm{~g}$, $16.25 \mathrm{~g}$, and $19.5 \mathrm{~g}$, with an error of $\pm 0.005 \mathrm{~g}$.

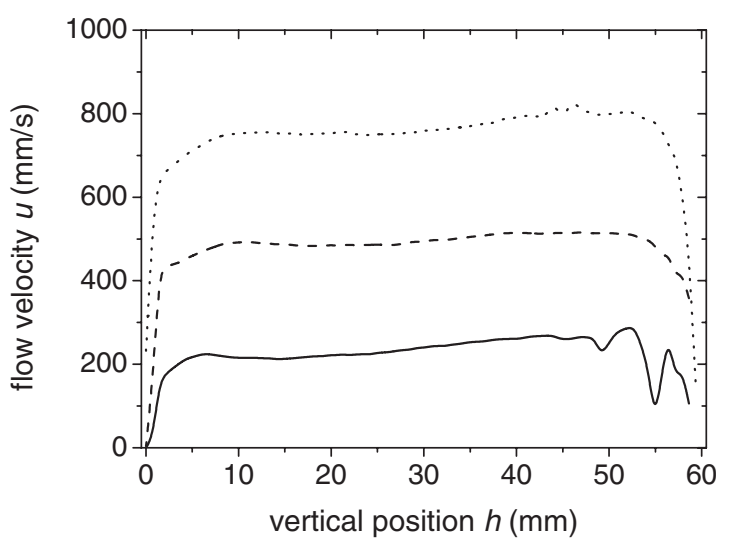

FIG. 3. Horizontal component $u$ of the flow velocity as a function of the vertical position $h$ for three different Reynolds numbers: $\mathrm{Re}=13000$ (solid line), $\mathrm{Re}=28500$ (dashed line), and $\mathrm{Re}=44000$ (dotted line).

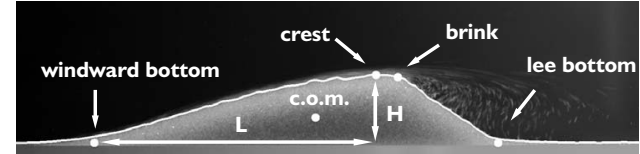

FIG. 4. A zoom to the picture at $t=10 \mathrm{~s}$ in Fig. 1: The found border of the barchan is marked white, the five striking points of a barchan are shown as dots, and the length $L$ and the height $H$ are plotted as double arrows.

For the classification of the flow velocity in the $6 \mathrm{~mm}$ wide channel we use an ultrasonic Doppler velocimeter (Signal Processing SA). This device measures the vertical velocity profiles as shown in Fig. 3. We conclude from the velocity profiles in Fig. 3 that the flow is fully turbulent with a distinct boundary layer and an almost constant value in the center of the channel. From these profiles we extract the mean flow velocity $\bar{u}$ with its standard deviation by averaging $u$ between the vertical positions $h=10 \mathrm{~mm}$ and $h$ $=50 \mathrm{~mm}$. For calculating the Reynolds number we use $\bar{u}$, the height of the channel $(60 \mathrm{~mm})$ and the kinematic viscosity $\nu=1 \mathrm{~mm}^{2} / \mathrm{s}$ of water at a temperature of $21.8 \pm 0.8^{\circ} \mathrm{C}$.

From pictures such as Fig. 1 and Fig. 4 the height profile of the barchan is extracted by finding the lowest gradient from dark to bright. In Fig. 4 the border found is marked in white. We also indicate five white dots which represent the characteristic positions of a barchan: windward bottom, crest, brink, lee bottom, and the center of mass (c.o.m.). The centroid of the two-dimensional border line indicates the

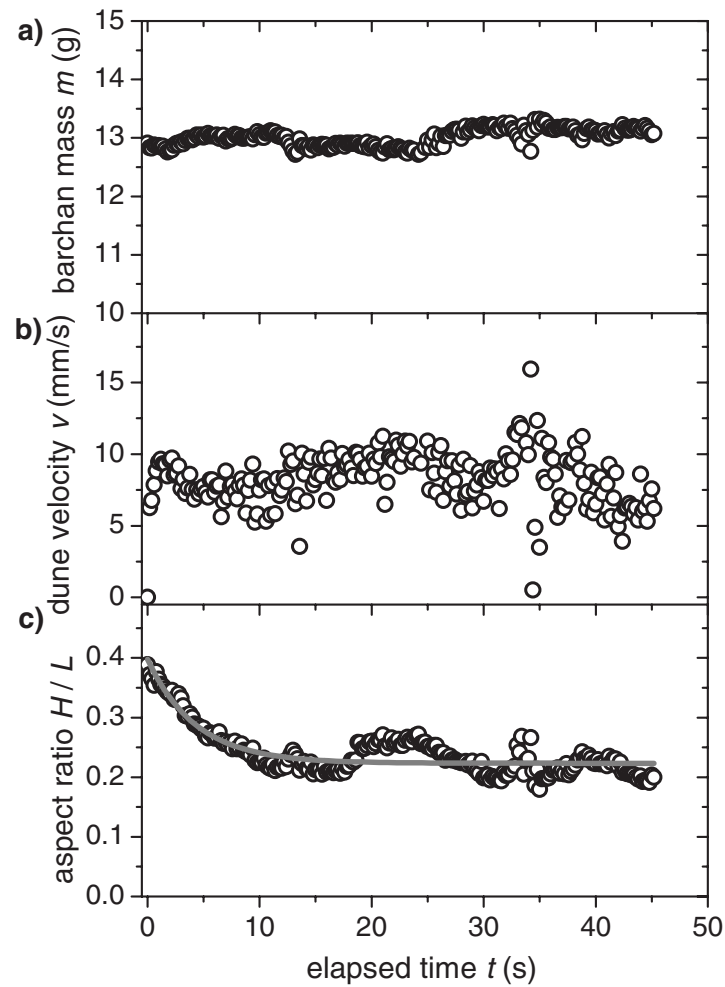

FIG. 5. The time evolution of the mass $m$, the velocity $v$ of the center of mass and the temporal evolution of the aspect ratio $H / L$ for the barchan dune in Fig. 1: The solid line is an exponential fit according to Eq. (1). 


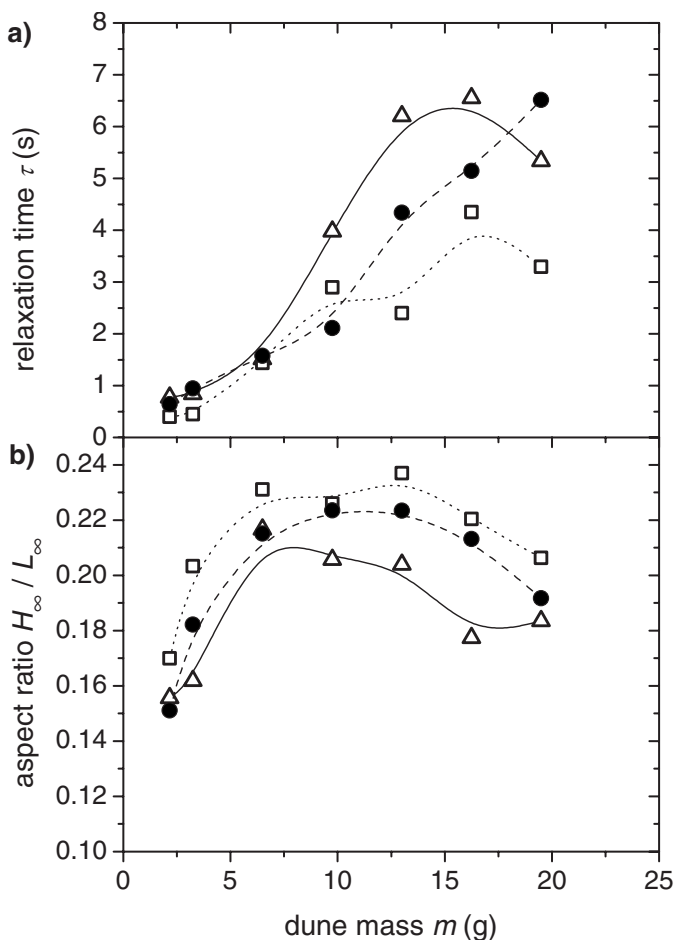

FIG. 6. Final aspect ratio $H_{\infty} / L_{\infty}$ and relaxation time $\tau$ for different barchan sizes and three different flow velocities: Re $=25500$ (triangles), $\operatorname{Re}=27000$ (squares), and $\mathrm{Re}=28500$ (circles). The dotted, dashed, and solid lines are guides to the eyes.

center of mass of the barchan. The other four points are determined by using the first and the second derivative of the smoothed height profile, obtained by utilizing a Gaussian filter. The crest is located at the highest point of the dune. The brink lies at the maximum of the second derivative. The windward and lee bottom lie at the minima of the second derivative.

Figure 5(a) shows the temporal evolution of the mass $m$ of the barchan dune in Fig. 1. The mass $m$ is obtained from cross sectional area, the width of the channel, and the density

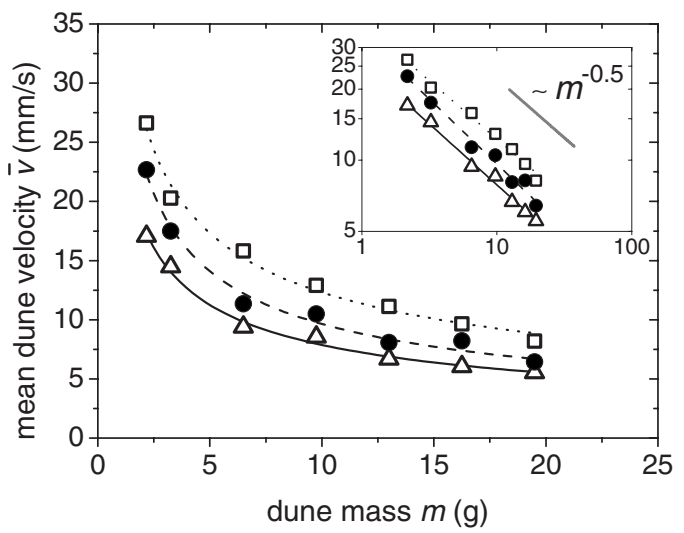

FIG. 7. Relation between mean barchan velocity $\bar{v}$ and the mass of the barchan dune $m$ for three different flow velocities: Re $=25500$ (triangles), $\operatorname{Re}=27000$ (squares), and $\mathrm{Re}=28500$ (circles). The dotted, dashed, and solid lines are fits to $\bar{v} \sim m^{-\alpha}$. The inset shows the same data on double-logarithmic scales.
TABLE I. Fit parameters obtained from the experimental data shown in Fig. 7 and Fig. 8.

\begin{tabular}{lccc}
\hline \hline $\operatorname{Re}$ & $\alpha$ & $\beta$ & $\gamma$ \\
\hline 25500 & $0.51 \pm 0.02$ & $0.81 \pm 0.05$ & $1.00 \pm 0.06$ \\
27000 & $0.55 \pm 0.03$ & $0.77 \pm 0.04$ & $1.02 \pm 0.07$ \\
28500 & $0.49 \pm 0.02$ & $0.73 \pm 0.04$ & $0.89 \pm 0.06$ \\
\hline \hline
\end{tabular}

of the glass beads. The temporal evolution of the horizontal velocity $v$ of the center of mass is plotted in Fig. 5(b). It serves to define the mean dune velocity $\bar{v}$.

As the snapshots in Fig. 1 show, the barchan dune becomes lower and longer during the measure time until it reaches a steady state. This is the case when the ratio $H / L$ becomes constant. The height $H$ and the length $L$ are determined as shown in Fig. 4. To obtain a quantitative statement about the steady state, we fit the empirical function

$$
\frac{H}{L}=\frac{H_{\infty}}{L_{\infty}}+a e^{-t / \tau}
$$

to the temporal evolution of $H / L$.
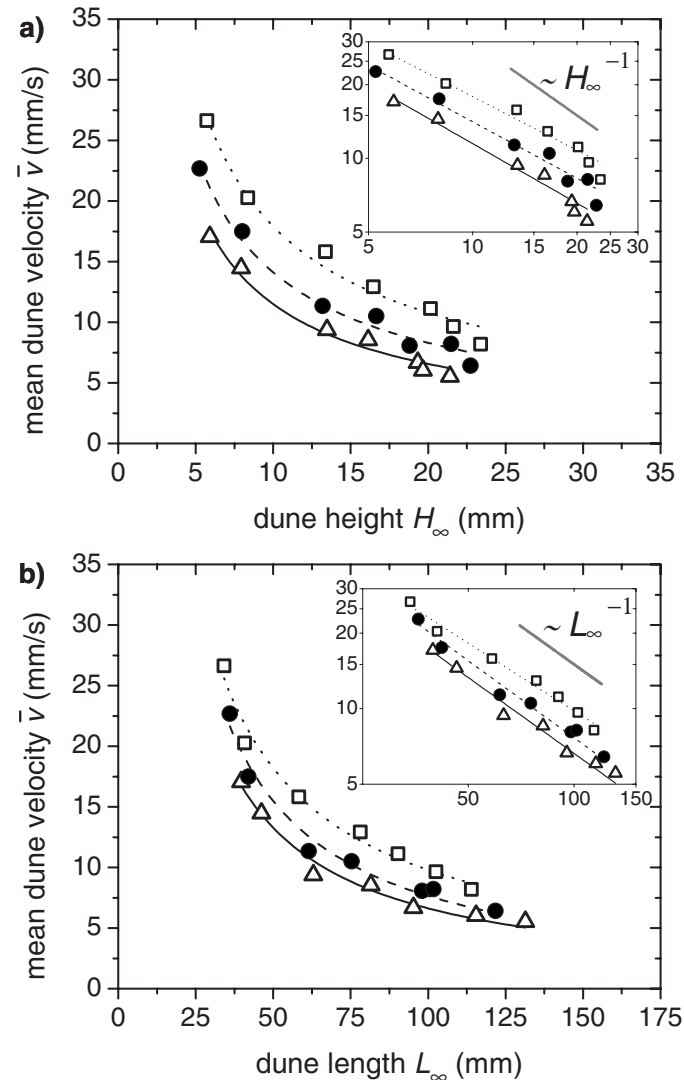

FIG. 8. Relation between mean barchan velocity $\bar{v}$ and height $H_{\infty}$, respectively, length $L_{\infty}$ for three different flow velocities: Re $=25500$ (triangles), $R e=27000$ (squares), and $R e=28500$ (circles). The dotted, dashed, and solid lines are fits to $\bar{v} \sim H_{\infty}^{-\beta}$, respectively, $\bar{v} \sim L_{\infty}^{-\gamma}$. The insets show the same data within double-logarithmic plots. 
The data points and the fit are shown in Fig. 5(c). The attractor is represented by the offset $H_{\infty} / L_{\infty}$ of Eq. (1). The aspect ratio $a$ describes the deviation of the initial aspect ratio from its steady-state value $H_{\infty} / L_{\infty}$. The parameter $\tau$ is the relaxation time for a triangle heap to achieve the stable shape of a steady-state barchan dune, as discussed in the theoretical model by Kroy et al. [12].

The dependence of the final aspect ratio $H_{\infty} / L_{\infty}$ and the relaxation time $\tau$ on the size of the barchan dunes is shown in Fig. 6. It is obvious that $H_{\infty} / L_{\infty}$ does not have the same value for different flow velocities and different barchan dune sizes, an important fact which confirms the theoretical models in Refs. [12,14,30].

Figure 6(a) shows $\tau$ versus the size, respectively, as a function of the mass of different barchan dunes for three different flow velocities. The time $\tau$ increases with the size of the barchan dune and decreases with the flow velocity.

In Fig. 7 the mean dune velocity is plotted versus the mass of the barchan dune. We fit our experimental data to the function $\bar{v} \sim m^{-\alpha}$. The resulting values for $\alpha$ are shown in Table I. For the mean value we can assume $\alpha \approx 0.5$ for all three flow velocities as predicted by the two-dimensional model by Andreotti et al. [14].

Figure 8 shows the relations between dune velocity and $H_{\infty}$, respectively, $L_{\infty}$ for three different Reynolds numbers. To obtain a quantitative relation we use the functions $\bar{v}$ $\sim H_{\infty}^{-\beta}$ and $\bar{v} \sim L_{\infty}^{-\gamma}$ as fits to the data points.

Table I shows the results for the exponents $\beta$ and $\gamma$. From these results we can assume that $\beta=0.8$ and $\gamma=1.0$ within the errors of measurement.

According to ideas dating back to Bagnold's work it can be inferred from mass conservation for a barchan dune that the velocity of the dune is related to the flux of grains $Q$ and the height $H_{\infty}$ by $\bar{v}=Q H_{\infty}^{-1}$ [20]. Relation $\bar{v} \sim H_{\infty}^{-1}$ follows if the flux is independent of $H_{\infty}$. The statement of the independent flux strictly holds only if the steady-state shape and aspect ratio are scale invariant, which is true for large dunes where deviations from scale invariance are not very pronounced.
However, general considerations and numerical simulations have shown that the scaling of the migration velocity with dune size for roughly similar shapes scale rather as $\bar{v}$ $\sim L_{\infty}^{-1}$ for moderate winds and small dunes [12]. The difference between these predictions is only noticeable for small dunes. The presently available field data are not accurate enough to clearly distinguish between $\bar{v} \sim H_{\infty}^{-1}$ and $\bar{v} \sim L_{\infty}^{-1}$. Our experiment seems to favor the theory of Kroy et al. [12] over the height scaling $\bar{v} \sim H_{\infty}^{-1}$ because the measured exponent $\gamma$ for the length scaling is closer to 1 .

Some open questions remain. For example, it is not yet clear whether the overall height of the channel determining the height of the driving fluid layer has a major influence on the size, shape, or speed of the model barchan dunes. Preliminarily investigations with $6 \mathrm{~mm}$ wide inserts of different heights show that, below a channel height of $30 \mathrm{~mm}$, the mean dune velocity increases dramatically whereas above this threshold value the variation of the velocity is in the range of $10 \%$. We interpret this as a hint that the dynamics of the observed barchan dunes depends only weakly on the absolute height of the channel.

To conclude, we designed an experiment that allows the quantitative measurement of the shape and the dynamics of a two-dimensional cut of three-dimensional barchan dunes found in nature. The dynamical system of a barchan dune evolves after a short relaxation time from a symmetric heap to a steady-state solution with a constant aspect ratio of height to length. It can be shown experimentally that the resulting steady-state shapes for barchan dunes of different sizes are not scale invariant. Consequentially, height and length scales for different dune sizes must be treated independently. Notably these results are in agreement with existing minimal models and simulations. Especially the predicted relation $\bar{v} \sim L_{\infty}^{-1}$ applies to the barchan dunes in our experiments.

We are grateful for support from Deutsche Forschungsgemeinschaft through Contracts No. Ak13/12-1 and No. Kr1877/3-1 (Forschergruppe 608 'Nichtlineare Dynamik komplexer Kontinua').
[1] IPCC, Climate Change 2007: The Physical Science Basis (Cambridge University Press, Cambridge, 2007).

[2] K. Pye and H. Tsoar, Aeolian Sand and Sand Dunes (Unwin Hyman, London, 1990).

[3] N. Lancaster, Geomorphology of Desert Dunes (Routledge, London, 1995).

[4] Aeolian Environments, Sediments and Landforms, edited by A. S. Goudie, I. Livingston and S. Stokes (Wiley, Chichester, 1995).

[5] S. Mitha, M. Q. Tran, B. T. Werner, and P. K. Haff, Acta Mech. 63, 267 (1986).

[6] J. C. R. Hunt, S. Leibovich, and K. J. Richards, Q. J. R. Meteorol. Soc. 114, 1435 (1988).

[7] W. S. Weng, J. C. R. Hunt, D. J. Carruthers, A. Warren, C. F. S. Wiggs, I. Livingstone, and I. Castro, Acta Mech. 2, 1 (1991).
[8] P. S. Jackson and J. C. R. Hunt, Q. J. R. Meteorol. Soc. 101, 925 (1975).

[9] I. Livingstone, G. F. S. Wiggs, and C. M. Weaver, Earth-Sci. Rev. 80, 239 (2007).

[10] G. Sauermann, P. Rognon, A. Poliakov, and H. J. Herrmann, Geomorphology 36, 47 (2000).

[11] H. J. Herrmann and G. Sauermann, Physica A 283, 24 (2000).

[12] K. Kroy, G. Sauermann, and H. J. Herrmann, Phys. Rev. Lett. 88, 054301 (2002); Phys. Rev. E 66, 031302 (2002).

[13] V. Schwämmle and H. J. Herrmann, Nature (London) 426, 619 (2003).

[14] B. Andreotti, P. Claudin, and S. Douady, Eur. Phys. J. B 28, 321 (2002); 28, 341 (2002).

[15] E. J. R. Parteli and H. J. Herrmann, Phys. Rev. Lett. 98, 198001 (2007)

[16] H. Elbelrhiti, B. Andreotti, and P. Claudin, Nature (London) 
437, 720 (2005).

[17] H. Elbelrhiti, B. Andreotti, and P. Claudin, J. Geophys. Res. 113, F02S15 (2008).

[18] B. R. White and H. Mounla, Acta Mech. 1, 145 (1991).

[19] E. J. R. Parteli, O. Durán, and H. J. Herrmann, Phys. Rev. E 75, 011301 (2007).

[20] R. A. Bagnold, The Physics of Blown Sand and Desert Dunes (Chapman and Hall, London, 1941).

[21] K. Kroy and X. Guo, Phys. Rev. Lett. 93, 039401 (2004).

[22] P. Hersen, S. Douady, and B. Andreotti, Phys. Rev. Lett. 89, 264301 (2002).

[23] G. Sauermann, K. Kroy, and H. J. Herrmann, Phys. Rev. E 64, 031305 (2001).
[24] B. Andreotti and P. Claudin, Phys. Rev. E 76, 063301 (2007).

[25] E. J. R. Parteli, O. Durán, and H. J. Herrmann, Phys. Rev. E 76, 063302 (2007).

[26] F. Charru, Phys. Fluids 18, 121508 (2006).

[27] K. Kroy, S. Fischer, and B. Obermayer, J. Phys.: Condens. Matter 17, S1229 (2005).

[28] N. Endo, K. Taniguchi, and A. Katsuki, Geophys. Res. Lett. 31, L12503 (2004).

[29] N. Endo, T. Sunamura, and H. Takimoto, Earth Surf. Processes Landforms 30, 1675 (2005).

[30] S. Fischer, M. E. Cates, and K. Kroy, Phys. Rev. E 77, 031302 (2008). 